Cohen says. But as soon as they realized it was working, they would celebrate, "dancing and running around the room".

In their exuberance, they were letting the light from a desk lamp shine right onto the microscope. "We were actually recording our excitement," says Daniel Hochbaum, then a graduate student in Cohen's group. They toned down their celebrations, and a year later, the team published its study ${ }^{1}$ - one of the first to show that a fluorescent protein engineered into specific mammalian neurons could be used to track individual electric impulses in real time.

Neuroscientists have tried for decades to observe the swift electrical signals that are a major component of the brain's language. Although electrodes, the workhorse for measuring voltage, can reliably record the activity of individual neurons, they struggle to capture the signals of many, particularly for prolonged periods. But in the past two decades, scientists have found a way to embed fluorescent, voltageindicating proteins right into the cell membranes of neurons. With the right kind of microscope, they can then see cells lighting up as they talk to each other - be it in a whisper or a shout. Voltage imaging can also record electrical chatter between many neurons at once, and then average those signals across large chunks of brain tissue. This helps researchers to study the brain's electrical activity across different spatial scales, by listening not only to the voices of individual cells but also to "the roar of the crowd", Cohen says.

In the past 5 years, scientists have published about 1,000 papers on the topic, and major funding schemes such as the US National Institutes of Health's BRAIN initiative have sped up the development of new types of genetically engineered voltage indicators. In the hope of finding better variants, some groups have come up with strategies to screen millions of proteins for desired characteristics such as brightness. One such approach has identified an indicator that's twice as bright as similar sensors developed just four years earlier².

As these proteins improve, and advances in microscopy make it easier to see them, scientists hope to illuminate neuroscience's biggest puzzle: how the brain's cells work together to transform a system of electrical pulses into thoughts, actions and emotions. Researchers are still struggling to catch the full range of activity and to devise ways to see nerves firing fast and deep within brain tissue. But if advances can solve these technical challenges, "it would be revolutionary", says Rafael Yuste, who studies the function of neural circuits at Columbia University in New York City.

\section{HIGH-SPEED PROCESS}

The average human brain contains about 120 billion neurons, which constantly receive and send information through branch-like appendages called dendrites. Chemical or electrical signals that reach the dendrites produce small voltage changes across the cell's membrane, which are routed to the cell body. When the sum of the voltage changes reaches a point of no return, called a threshold, the neuron fires a large electrical spike - an action potential. This jolt whizzes at speeds of up to 150 metres per second along a neuronal branch, known as an axon, to another set of branching appendages. Here, chemical or electrical signals pass the information on to the next set of dendrites.

Neuronal signals converge, diverge and synchronize to produce a symphony of thoughts, emotions, actions and reactions, from the flush of a face to a baby's hiccup. But scientists' listening tools are extremely limited. First developed in the 1940s, miniature electrodes as thin as a hair can be inserted into the brain, up against or inside neurons, where they measure membrane voltage with precision and speed. But this approach can be used to monitor just one or a handful of neurons at once - and only for a limited amount of time, because the electrodes eventually damage the cell. It's like trying to get the gist of an orchestral arrangement by following one player for a few seconds.

Bundles of micro-electrodes can record the electrical activity of up to 200 cells at once, but because these electrodes are placed near to neurons rather than inside them, they can detect only the action potentials, the sharpest spikes in electrical activity. They are deaf to softer notes - the small electrical changes that do not push the neuron

\section{FLAYOURS OF FLUORESGENGE}

Scientists have built different types of genetically encoded voltage indicator

(GEVI). One major category (top) uses a membrane-bound portion of a

voltage-sensing protein, such as a sodium channel, fused to one or more

fluorescent proteins. Another category uses an opsin protein, such as a

microbial rhodopsin, a membrane channel that directly changes its

fluorescent properties in response to an electric field.
Voltage-sensing fusion protein

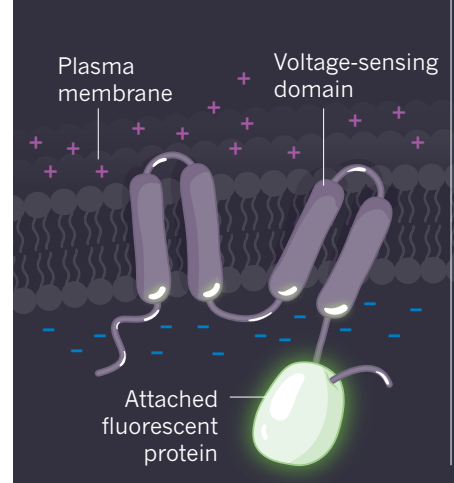

Opsin-based voltage indicator

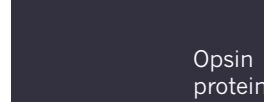

protein

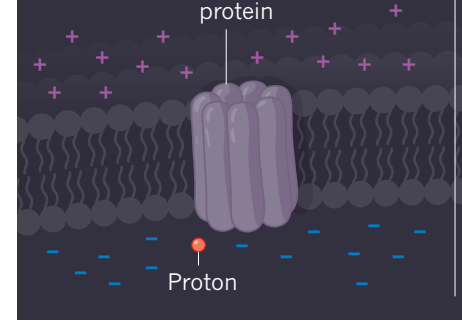

A voltage change across the membrane causes the GEVI to change shape, decreasing the fluorescence of the attached protein.

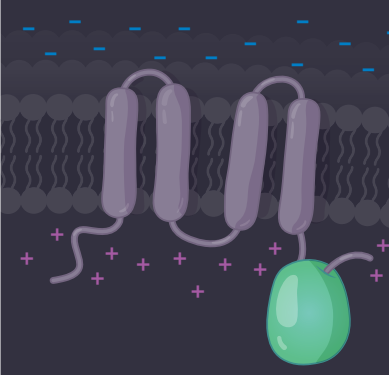

A voltage change across the membrane can help to add a proton to retinal, the light-sensitive portion of the opsin, which alters its fluorescence.

all the way to an action potential. These sub-threshold voltage changes are key to brain function, because they gradually add up to determine whether or not a neuron will fire.

In the hope of measuring quieter brain activity in larger populations of cells, scientists in the 1960s began toying with the idea of a sensor or probe that fluoresces in response to an electrical signal. The most popular probes, called calcium indicators, light up when they bind to calcium, which flows into the neuron as a result of a spike in electrical activity. But the technique, known as calcium imaging, provides only a proxy; it doesn't directly record membrane voltage. And although it will show the signal of big events such as action potentials, it misses things that are crucial for brain function, such as subtle swings in membrane voltage or the electric signals that inhibit action potentials. Imagine being able to hear only a burst of applause after a symphony concert: it's clear that the orchestra has performed, but what it was playing is anyone's guess.

In the 1970s, scientists started to develop dye sensors that detect changes in membrane voltage directly. The first versions of these dyes had to be painted onto the brain indiscriminately, so they labelled all cell types, including non-neuronal cells, making it difficult to parse the activity of specific neurons.

Then, in the 1990s, researchers started testing indicators that could be genetically engineered to show up only in neurons of interest. The first $^{3}$ genetically encoded voltage indicator (GEVI) was developed in 1997; since then, scientists have churned out more than two dozen sensors $^{4}$. Some of these are made by combining a voltage-sensitive protein with fluorescent molecules (see 'Flavours of fluorescence'). When these proteins detect a change in voltage, they change their 3D structure and alter the fluorescence of the molecule they're coupled to. Other voltage indicators are mutated versions of microbial rhodopsins, fluorescent molecules that cause a change in voltage across the plasma membrane 
in response to light. These proteins can also work in reverse, changing their response to light - and thus their fluorescence - in response to a change in membrane voltage.

\section{ALL IN THE DETAIL}

So far, GEVIs have proved successful in tracking individual action potentials in both cultured neurons, grown in a dish, and in the intact brains of a wide range of animals, from insects ${ }^{5}$ to mice ${ }^{6}$. One of the biggest promises of the technique is its potential to record not only the big events but also the small, sub-threshold changes in membrane voltage that reflect the messages that a neuron receives from neighbouring cells, Cohen says. "Voltage imaging lets you see the inputs to the neurons in vivo, which we had no way to look at previously," he says.

In the past year, Cohen and his colleagues developed new GEVIs and improved microscopy techniques to record such sub-threshold voltage changes from many neurons at once, including in the mouse brain ${ }^{7,8}$. The team was also able to record the electrical activity of the same neurons up to a week later. The ability to know exactly which neurons are being recorded and to keep track of them over time allows researchers to look at the wiring between those neurons, says Ed Boyden, a neuroscientist at the Massachusetts Institute of Technology in Cambridge. By doing so, "you can link the structure of the brain with its function", he says. "That's one of the core questions in all neuroscience."

Another advantage of GEVIs is that, unlike electrodes, which record mainly signals from the cell body, they can record electrical signals from any part of a nerve cell, right down to the tips of dendrites. That's like being able to listen specifically to the notes played by a pianist's left hand. "This is something that I've been dreaming for a long time - and I'm not alone," says Katalin Toth, a neurobiologist at Laval University in Quebec City, Canada. Many neuroscientists are striving to follow voltage across entire neurons to see how it changes in different regions of the cell, she says.

Wei Wei, a neurobiologist at the University of Chicago, Illinois, is using GEVIs to work out how different electrical inputs are integrated in the neurons of the mouse retina. Wei is interested in a class of neuron that responds more strongly to a visual stimulus when it is moving in a particular direction. By looking at how membrane voltage changes in different parts of these neurons, she hopes to understand how the cells sum up incoming signals to detect the direction of the movement.

Neurophysiologist Vincent Villette at the Ecole Normale Supérieure in Paris plans to use voltage sensors to study how regular fluctuations of sub-threshold electric signals determine how neurons in the mouse cerebellum coordinate muscle activity. "There's a lot to be understood on how cells act together," Villette says.

Getting a visual read-out of membrane voltage also allows scientists to see electrical signals that inhibit neuronal firing rather than trigger it. Because inhibitory signals are impossible to record with approaches such as calcium imaging, it's unclear how exactly they shape brain activity, says Rosa Cossart, a neurobiologist at the Mediterranean Institute of Neurobiology in Marseilles, France.

Cossart has been using electrodes and calcium imaging for years, but she's now eager to try GEVIs. She hopes these sensors will allow her to measure voltage at high speed across multiple neurons - at least 50 - at the same time in a living mouse. This would help to understand how groups of neurons integrate electrical signals - both excitatory and inhibitory - to support activities that are crucial for brain development and function, she says.

\section{DEEP CHALLENGES}

Despite the high expectations, getting GEVIs to work in the laboratory can be a hassle. Take Helen Yang: as a graduate student at California's Stanford University, she decided to try GEVIs as a way to study neurons in the fruit fly's visual system. But peering through the microscope during her first experiment, Yang saw no change in the cells' fluorescence, not even when she flashed a bright light in the flies' eyes. It wasn't until she analysed the data that she realized that the visual stimuli were producing a signal, it was just a tiny one. "I was pretty excited, but my lab-mates were less so," she says. "The responses were pretty small and noisy."

Yang started to play with the microscope settings, increasing the laser power and speeding up the imaging. "I basically made it go as fast as our microscope could," she says. That's because the indicator's response to an electrical signal was so fast that the change in fluorescence was detectable just for a fraction of a second. "If you're only capturing one frame during the time that the cell is responding, the response doesn't look big at all," Yang says.

Yang eventually managed to use GEVIs to investigate how the flies' neurons process visual cues ${ }^{5}$, but the sorts of challenges she faced have so far prevented voltage imaging from becoming a mainstream technique. It requires advanced, often custom-built microscope platforms, Cohen says. "You can't just do this on your grandmother's fluorescent microscope."

In the past five years, financial support from the BRAIN initiative has boosted advances in the field, including the development of better GEVIs, says Michael Lin, a protein engineer at Stanford.

In parallel with the development of new sensors, scientists are working on techniques to image with precision the fast electrical signals travelling through the brain. One challenge is that most of the available techniques work well only with cells in a dish or on the surface of the brain. But the mammalian brain isn't transparent: in fact, it looks like tofu, says $\mathrm{Na}$ Ji, a physicist at University of California, Berkeley.

To peer deeper, researchers have to turn to moreinvasive methods, such as removing some of the overlying tissue or sticking tiny optical devices called micro-endoscopes directly into the brain. An alternative, non-invasive way to look into opaque tissues - up to 1 millimetre deep - is two-photon microscopy. This technique uses longer-wavelength, lower-energy light, which can penetrate deeper into tissues. Because two-photon microscopes illuminate and record from only a single spot at a time, they capture images too slowly to track much of the brain's fast chatter. But specialists are confident that advances in the technology will soon make it possible to see the signals produced by GEVIs at higher speed. "It's absolutely doable," Ji says.

If the different approaches can overcome these challenges, scientists have no doubt that voltage imaging will become a standard approach for measuring brain activity. "In the next year or two, we'll see a lot of papers that have applied voltage sensors and learned about biology," says Thomas Clandinin, a neurobiologist at Stanford. Some say that the technique might even replace electrodes for questions related to how neurons process and integrate information.

Early-career researchers are particularly optimistic: Hochbaum, who is now a postdoctoral fellow at Harvard Medical School in Boston, says that in the long term, GEVIs will be a go-to tool for studying how different compartments in the cell respond to sub-threshold signals. He plans to use voltage imaging to understand how such signals alter the connection between neurons, a key process in learning. The possibilities are exciting, Hochbaum says, but he's learnt at least one important lesson from those early days of jumping around the lab in glee after seeing a glow in a microscope: when the experiments work, keep the celebrations to a minimum.

Giorgia Guglielmi is a freelance science journalist in Cambridge, Massachusetts.

1. Kralj, J. M., Douglass, A. D., Hochbaum, D. R., Maclaurin, D. \& Cohen, A. E. Nature Meth. 9, 90-95 (2012)

2. Piatkevich, K. D. et al. Nature Chem. Biol. 14, 352-360 (2018)

3. Siegel, M. S. \& Isacoff, E. Y. Neuron 19, 735-741 (1997)

4. Xu, Y., Zou, P. \& Cohen, A. E. Curr. Opin. Chem. Biol. 39, 1-10 (2017).

5. Yang, H. H. et al. Cell 166, 245-257 (2016).

6. Gong, Y. et al. Science 350, 1361-1366 (2015).

7. Adam, Y. et al. Preprint at bioRxiv https://doi.org/10.1101/281618 (2018)

8. Chien, M.-P. et al. Preprint at bioRxiv https://doi.org/10.1101/211946 (2017). 